\title{
Direct observation of oxygen configuration on individual graphene oxide sheets
}

Liu, Zilong; Nørgaard, Kasper; Overgaard, Marc H.; Ceccato, Marcel; Mackenzie, David M.A.; Stenger, Nicolas; Stipp, Susan L.S.; Hassenkam, Tue

\section{Published in:}

Carbon

Link to article, DOI:

10.1016/j.carbon.2017.10.100

Publication date:

2018

Document Version

Peer reviewed version

Link back to DTU Orbit

Citation (APA):

Liu, Z., Nørgaard, K., Overgaard, M. H., Ceccato, M., Mackenzie, D. M. A., Stenger, N., Stipp, S. L. S., \& Hassenkam, T. (2018). Direct observation of oxygen configuration on individual graphene oxide sheets. Carbon, 127, 141-148. https://doi.org/10.1016/j.carbon.2017.10.100

\section{General rights}

Copyright and moral rights for the publications made accessible in the public portal are retained by the authors and/or other copyright owners and it is a condition of accessing publications that users recognise and abide by the legal requirements associated with these rights.

- Users may download and print one copy of any publication from the public portal for the purpose of private study or research.

- You may not further distribute the material or use it for any profit-making activity or commercial gain

- You may freely distribute the URL identifying the publication in the public portal 


\section{Accepted Manuscript}

Direct observation of oxygen configuration on individual graphene oxide sheets

Zilong Liu, Kasper Nørgaard, Marc H. Overgaard, Marcel Ceccato, David Mackenzie, Nicolas Stenger, Susan L.S. Stipp, Tue Hassenkam

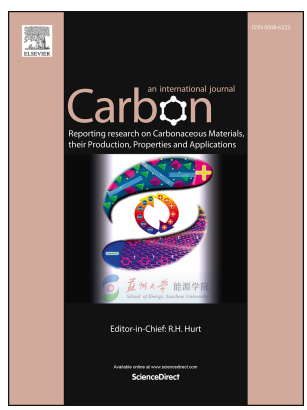

PII: S0008-6223(17)31093-X

DOI: $\quad$ 10.1016/j.carbon.2017.10.100

Reference: CARBON 12528

To appear in: Carbon

Received Date: 13 July 2017

Revised Date: 27 October 2017

Accepted Date: 28 October 2017

Please cite this article as: Z. Liu, K. Nørgaard, M.H. Overgaard, M. Ceccato, D. Mackenzie, N. Stenger, S.L.S. Stipp, T. Hassenkam, Direct observation of oxygen configuration on individual graphene oxide sheets, Carbon (2017), doi: 10.1016/j.carbon.2017.10.100.

This is a PDF file of an unedited manuscript that has been accepted for publication. As a service to our customers we are providing this early version of the manuscript. The manuscript will undergo copyediting, typesetting, and review of the resulting proof before it is published in its final form. Please note that during the production process errors may be discovered which could affect the content, and all legal disclaimers that apply to the journal pertain. 


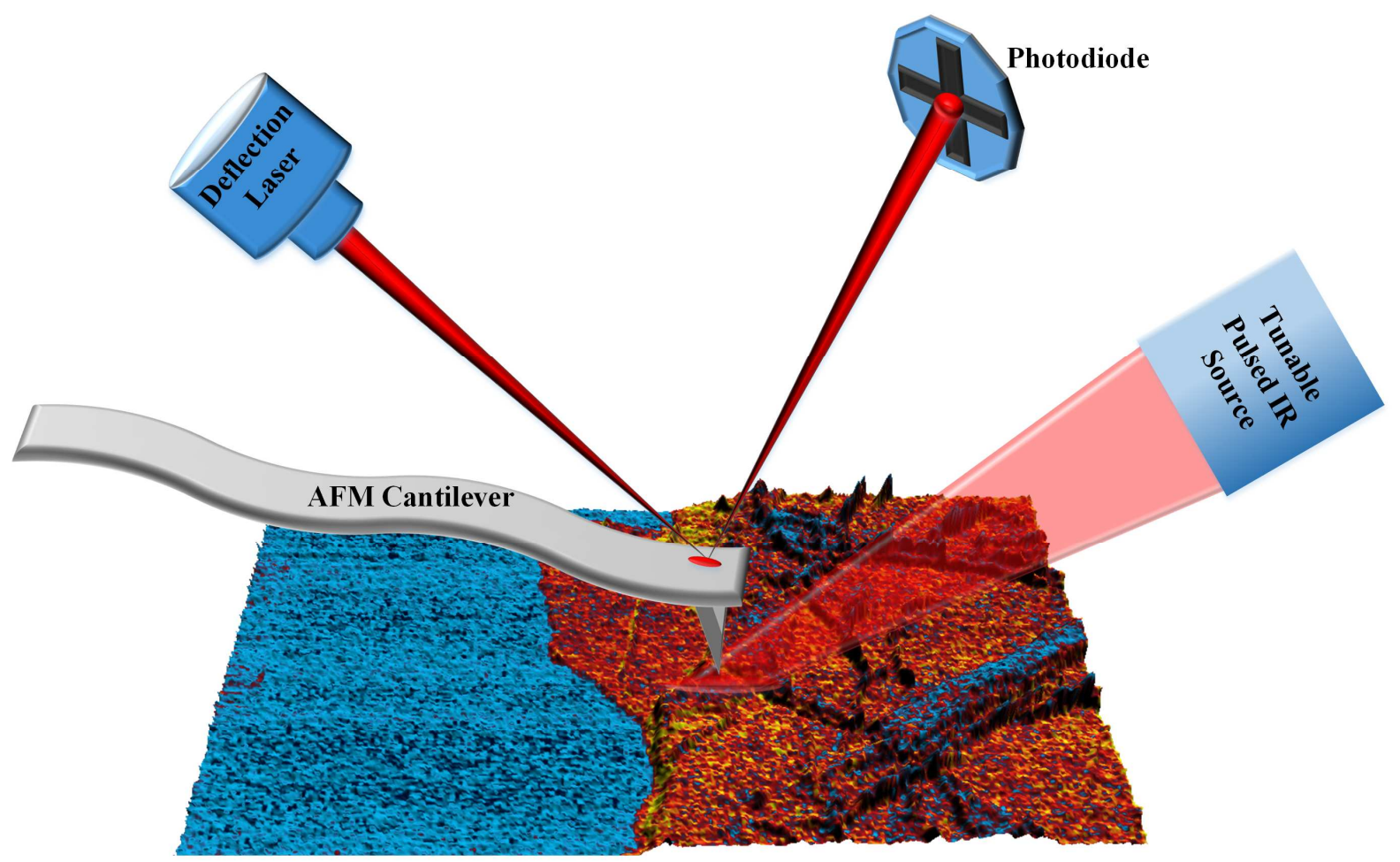

Graphene oxide on Au substrate 


\section{Direct Observation of Oxygen Configuration on Individual Graphene Oxide Sheets}

Zilong Liu ${ }^{\mathrm{a}}$, Kasper Nørgaard ${ }^{\mathrm{a}}$, Marc H. Overgaard ${ }^{\mathrm{a}}$, Marcel Ceccato ${ }^{\mathrm{a}}$, David M.A.

Mackenzie $^{\mathrm{b}}$, Nicolas Stenger ${ }^{\mathrm{c}}$, Susan L.S. Stipp ${ }^{\mathrm{a}}$, Tue Hassenkam ${ }^{\mathrm{a}, *}$

${ }^{a}$ Nano-Science Center, Department of Chemistry, University of Copenhagen,

Universitetsparken 5, 2100 Copenhagen, Denmark

${ }^{\mathrm{b}}$ Center for Nanostructured Graphene (CNG), Department of Micro- and Nanotechnology, Technical University of Denmark, Ørsteds Plads 345 B, Kongens Lyngby 2800, Denmark

${ }^{\mathrm{c}}$ Center for Nanostructured Graphene (CNG), Department of Photonics, Technical University of Denmark, Ørsteds Plads 345 A, Kongens Lyngby 2800, Denmark

*Corresponding author: Tue Hassenkam, Tel: +45 26552030, E-email: tue@ nano.ku.dk 


\section{Abstract}

Graphene oxide (GO) is an interesting material that has the potential for a wide range of applications. Critical for these applications are the type of oxygen bond and its spatial distribution on the individual GO sheets. This distribution is not yet well understood. Few techniques offer a resolution high enough to unambiguously identify oxygen configuration. We used a new, label free spectroscopic technique to map oxygen bonding on GO, with spatial resolution of nanometres and high chemical specificity. AFM-IR, atomic force microscopy coupled with infrared spectroscopy, overcomes conventional IR diffraction limits, producing IR spectra from specific points as well as chemical maps that are coupled to topography. We have directly observed oxygen bonding preferentially on areas where graphene is folded, in discrete domains and on edges of GO. From these observations, we propose an updated structural model for $\mathrm{GO}$, with $\mathrm{C}=\mathrm{O}$ on its edge and plane, which confirms parts of earlier proposed models. The results have interesting implications. Determining atomic position and configuration from precise imaging offers the possibility to link nanoscale structure and composition with material function, paving the way for targeted tethering of ions, polymers and biomaterials. 


\section{Introduction}

One of the limitations for more extensive use of graphene-based materials is the challenge of mass production but graphene oxide (GO) has been widely adopted as a starting material for material synthesis ${ }^{[1,2]}$ and there is potential for many more technological applications, ${ }^{[3-6]}$ such as optoelectrical and electronic devices, heterogeneous catalysis, polymer composites as well as in biomedicine and biotechnology. Because GO is covalently decorated with oxygen on its plane and edges, ${ }^{[7]}$ it is hydrophilic, which makes fabrication of graphene containing devices from solution both economical and practical. Perhaps most important, the aromatic scaffolds and the oxygen networks on GO provide abundant anchoring sites for functionalization by ions, polymers, nanoparticles and biological species. ${ }^{[5,6]}$

In the aim of tailoring the fundamental properties of $\mathrm{GO}$, to unleash its potential for applications, detailed understanding of atomic composition and bonding is required. The ideal graphene structure, a single layer of $\mathrm{sp}^{2}$ bonded carbon atoms arranged in a honeycomb motif, is well known. However, once oxidized, GO restructures so the atomic arrangement becomes heterogeneous, affecting its composition and chemical properties. The unresolved questions are: What is the distribution of oxygen bonding on GO? And how does this affect the underlying carbon template? An accurate structural model is a prerequisite for predicting reactivity. Uncertainty remains because the ultrathin GO layers make analysis difficult. Several spectroscopic approaches have been used to investigate the type, abundance and distribution of oxygen that is chemically bonded to the carbon network. Such techniques include nuclear magnetic resonance (NMR) ${ }^{[7-9]}$ X-ray absorption near edge spectroscopy (XANES) ${ }^{[10]}$ Raman spectroscopy, ${ }^{[11]}$ X-ray photoelectron spectroscopy $(\mathrm{XPS}),{ }^{[12,13]}$ and Fourier transform infrared spectroscopy (FTIR). ${ }^{[12,14-16]}$ 
Based on data from NMR with isotope labelling, the most widely used structural model of GO was proposed by Lerf-Klinowski (LK) ${ }^{[7]}$ In this model, oxygen is bonded singly to the GO carbon plane, in configurations reminiscent of epoxy and hydroxyl, which provide structural rigidity to GO, while oxygen is bonded singly and doubly on the edges, as in carboxyl, and these oxidized groups give rise to its solubility in water. The general model has not changed much since it was proposed but more recent results ${ }^{[8]}$ have triggered modifications, such as addition of oxygen in other configurations, as in ketones, lactols and ester carbonyls. The model was further improved by the results from a set of experiments that revealed simple spatial connectivity between regions with oxygen in epoxide and hydroxyl arrangements. ${ }^{[9]}$ Use of other analytical techniques has also provided indirect evidence that is consistent with the LK model. ${ }^{[10,17-19]}$

With XANES, the various carbon bonding environments for oxygen were explored, to resolve the composition and structure of GO. ${ }^{[10]}$ Results confirmed the essence of the LK model but could only provide a macroscopic perspective, i.e. data averaged over many surfaces. In other studies, the oxidized and unoxidized areas of GO have been explored using high resolution transmission electron microscopy $(\mathrm{TEM})^{[17]}$ and electron energy loss spectroscopy (EELS), ${ }^{[18,19]}$ providing proof that oxygen atoms are randomly attached to GO sheets, converting carbon bonds from $\mathrm{sp}^{2}$ to $\mathrm{sp}^{3}$. Other spectroscopic techniques, ${ }^{[11-16]}$ mainly Raman, XPS and FTIR, have provided information about oxygen bonding neighbours and their relative proportions but details about the actual spatial distribution of oxygen and its bonding types on GO, at the nanometer scale, has not been possible.

Our purpose was to map the spatial distribution of $\mathrm{C}-\mathrm{O}$ and $\mathrm{C}=\mathrm{O}$ bonds on $\mathrm{GO}$ and use the direct experimental observations from the nanometre scale to propose a more robust structural model. We characterized GO and identified the location of oxygen and the type of 
bonding using a new technique: AFM-IR. ${ }^{[20-22]}$ It combines atomic force microscopy with a pulsed IR laser that is tuneable over the mid IR range $\left(900-3600 \mathrm{~cm}^{-1}\right)$. It allows IR spectroscopy at AFM resolution, namely tens of nanometres in $x$ and $y$ and $0.1 \mathrm{~nm}$ or less in $z$. This resolution is not possible with standard, optical IR because of the diffraction limit but by combining the best of two worlds, we can visualize oxygen bonding on GO with near molecular resolution.

\section{Experimental}

\subsection{Sample preparation}

Commercial graphite powder (Grade 3061) was obtained from Asbury Carbon Mills. $\mathrm{H}_{2} \mathrm{SO}_{4}$ (95-98\%), $\mathrm{KMnO}_{4}$ and $\mathrm{H}_{2} \mathrm{O}_{2}(33 \%)$ were obtained from Sigma Aldrich and $\mathrm{NaNO}_{3}$ was obtained from Merck. All were reagent grade or better and were used as received.

GO was prepared by harsh oxidation of the graphite powder by the modified Hummers method. ${ }^{[23,24]}$ In a typical reaction, we put $1 \mathrm{~g}$ graphite and $0.5 \mathrm{~g} \mathrm{NaNO}_{3}$ into a round bottomed flask and added $24 \mathrm{~mL}$ of concentrated $\mathrm{H}_{2} \mathrm{SO}_{4}$. The mixture was placed on ice and stirred for 5-10 minutes, then $6 \mathrm{~g} \mathrm{KMnO}_{4}$ was added in small portions over a period of 30 minutes. The reaction mixture was stirred on ice for 2 hours more. The flask was then transferred to an oil bath, preheated to $35{ }^{\circ} \mathrm{C}$ and left to oxidize for 16 hours. Reaction refinement and purification were initiated by adding $40 \mathrm{~mL}$ ultrapure deionised water dropwise into the reaction mixture under stirring, which was then briefly heated to $90{ }^{\circ} \mathrm{C}$ for 5 minutes. The reaction vessel was allowed to cool to room temperature and $100 \mathrm{~mL}$ ultrapure water was added in one portion. Oxidation was terminated by adding $4 \mathrm{~mL} \mathrm{H}_{2} \mathrm{O}_{2}(33 \%)$ dropwise into the flask. 
The freshly synthesized GO was rinsed 5 times with ultrapure water and centrifuged, to remove residual acid and salts. Typically, 4-6 repetitions were sufficient to achieve proper washing, which was tested by measuring $\mathrm{pH}$ of a neutral supernatant. The material was delaminated by mild sonication for 4 minutes and centrifugation at $2000 \mathrm{rpm}$ until no visual sedimentation was observed. Finally, the GO was centrifuged twice at $9000 \mathrm{rpm}$ to remove oxidative debris, ${ }^{[25]}$ which are small size functionalized graphene rich in oxidized groups. For each experiment, a $30 \mu \mathrm{L}$ aliquot of the GO solution was deposited on a clean gold substrate and dried in a stream of clean nitrogen gas.

\section{$2.2 X$-ray photoelectron spectroscopy}

The chemical state and the element composition of GO were determined with XPS (Kratos Axis Ultra DLD), using monochromatized $\mathrm{Al} K \alpha(h v=1486.6 \mathrm{eV})$ as the excitation source. ${ }^{[26]}$ The data were analyzed using commercial software, CasaXPS, and a Shirley background fit. The carbon peaks were referenced to $285.0 \pm 0.1 \mathrm{eV} \cdot{ }^{[13]}$ Uncertainty in XPS binding energy is about $0.1 \mathrm{eV}$. Uncertainty in the atomic percentages determined from XPS data is on the order of $5-10 \%$.

\subsection{Raman spectroscopy}

Raman spectroscopy was performed using a Thermo Scientific DXRxi fitted with a $532 \mathrm{~nm}$ laser. Using a laser power of $3 \mathrm{~mW}, 180 \mathrm{~Hz}$ collection frequency was scanned 900 times per pixel through at $100 \mathrm{x}$ objective.

\subsection{Fourier transform infrared spectroscopy}

We used a FTIR Vertex v70 from Bruker coupled to a infrared microscope Hyperion 2000 for micro-FTIR measurements. The light source is a $\mathrm{SiC}$ "glowbar" and we measured in reflection and transmission modes. The detector is a liquid nitrogen cooled MCT (Mercury 
Cadmium Telluride) detector. The measurement window was $900-2000 \mathrm{~cm}^{-1}$ and $2500-3600$ $\mathrm{cm}^{-1}$ range. We averaged over 128 spectra with a spectral resolution is $4 \mathrm{~cm}^{-1}$.

\subsection{Infrared nanospectroscopy using AFM-IR}

The localized nanoscale mid-IR spectra were collected using a nanoIR2 instrument (AFM-IR) from Anasys Instruments, Inc. It consists of an AFM with a pulsed, tunable IR laser. With AFM-IR, one can generate topographic images of GO with the AFM and it also serves as a nearfield detector for measuring the absorption of IR radiation. In mapping mode, one sets the laser at a specific wavelength and collects absorption intensity data over a selected area. Spatial resolution is about $40 \mathrm{~nm}$ per pixel. In spectra mode, one can acquire IR spectra at specific sites while ramping the energy of the laser.

With the tunable IR sources on our instrument, pulse length is about $10 \mathrm{~ns}$ and energy ranges over the middle IR region $\left(900-3600 \mathrm{~cm}^{-1}\right)$. In Supplementary Fig. S1A, it shows the principles of the technique based on the photothermal induced resonance effect. ${ }^{[20,21]}$ When IR radiation is absorbed by a sample, it heats, causing an almost instantaneous thermal expansion that induces oscillations of the AFM cantilever. The oscillations decay in a ringdown mode (Supplementary Fig. S1B), which typically contains multiple frequencies and amplitudes (Supplementary Fig. S1C). Because sample expansion is much faster than AFM feedback (typical laser repetition rate $1 \mathrm{kHz}$ ), it allows sufficient time for completing a ringdown and exciting resonant modes of the AFM cantilever before a new pulse arrives. The ringdown is usually analyzed by Fourier transform, to extract the useful information. The resonant amplitudes in the cantilever are proportional to the intensity of the IR radiation that was absorbed by the sample.

All of the AFM and IR spectroscopic images were obtained in contact mode. At the beginning of the IR spectra acquisition, IR background calibration was conducted in the 
ranges of 900-2000 and 2500-3600 $\mathrm{cm}^{-1}$, to provide a baseline for removing the effect of power variation in the IR source. Five background spectra were collected and averaged. The second mode of cantilever oscillation was chosen for the cantilever ringdown signal at a frequency center of $186 \mathrm{kHz}$, with a frequency window of $50 \mathrm{kHz}$. An average of 256 scans was used for optimization. We usually selected a low laser power to avoid burning the sample. The optimization step was repeated four times from a $800 \mu \mathrm{m}$ x $800 \mu \mathrm{m}$ search area to a 100 $\mu \mathrm{m} \times 100 \mu \mathrm{m}$ search area to optimize the signal and to centre it under the cross hair. We acquired the spectra over the $900-2000$ and $2500-3600 \mathrm{~cm}^{-1}$ ranges, with an energy resolution of $4 \mathrm{~cm}^{-1} / \mathrm{pt}$ and a co-average of 256 scans, which meant that it took a couple of minutes to record a spectrum. In the acquisition of IR maps, we reduced the scan rate to $0.1 \mathrm{~Hz}$ and the co-average to 16 scans. We took data at the frequency of 256 pixels in both $x$ and $y$. The total time for acquiring one map was $\sim 25$ minutes. The amplitude and frequency images were collected simultaneously by obtaining the contact resonant signal from the cantilever ringdown as a function of position.

\section{Results and Discussion}

Figure 1A is a topographic image of several flakes of GO adhering on a gold substrate. In the upper left corner (Sites 1,2 and 3), several layers (as many as six) of GO overlap, whereas in the upper right (Site 4), monolayer GO is visible. In the central to the lower right of the image (Sites 5 and 6), the bare gold surface is exposed. Six AFM-IR spectra were collected at these positions (Fig. 1B). All the positions were on basal planes far (at least $100 \mathrm{~nm}$ ) from any folds and edges in the GO. In the multilayer spectra (S1 to S3), one sharp peak occurs at $\sim 1025 \mathrm{~cm}^{-1}$ with small shoulders at $1010 \mathrm{~cm}^{-1}, 995 \mathrm{~cm}^{-1}$ and $975 \mathrm{~cm}^{-1}$. The dip at $\sim 1100 \mathrm{~cm}^{-1}$ is an artefact resulting from shift in laser power to increase instrument sensitivity above this wavenumber. 


\section{ACCEPTED MANUSCRIPT}
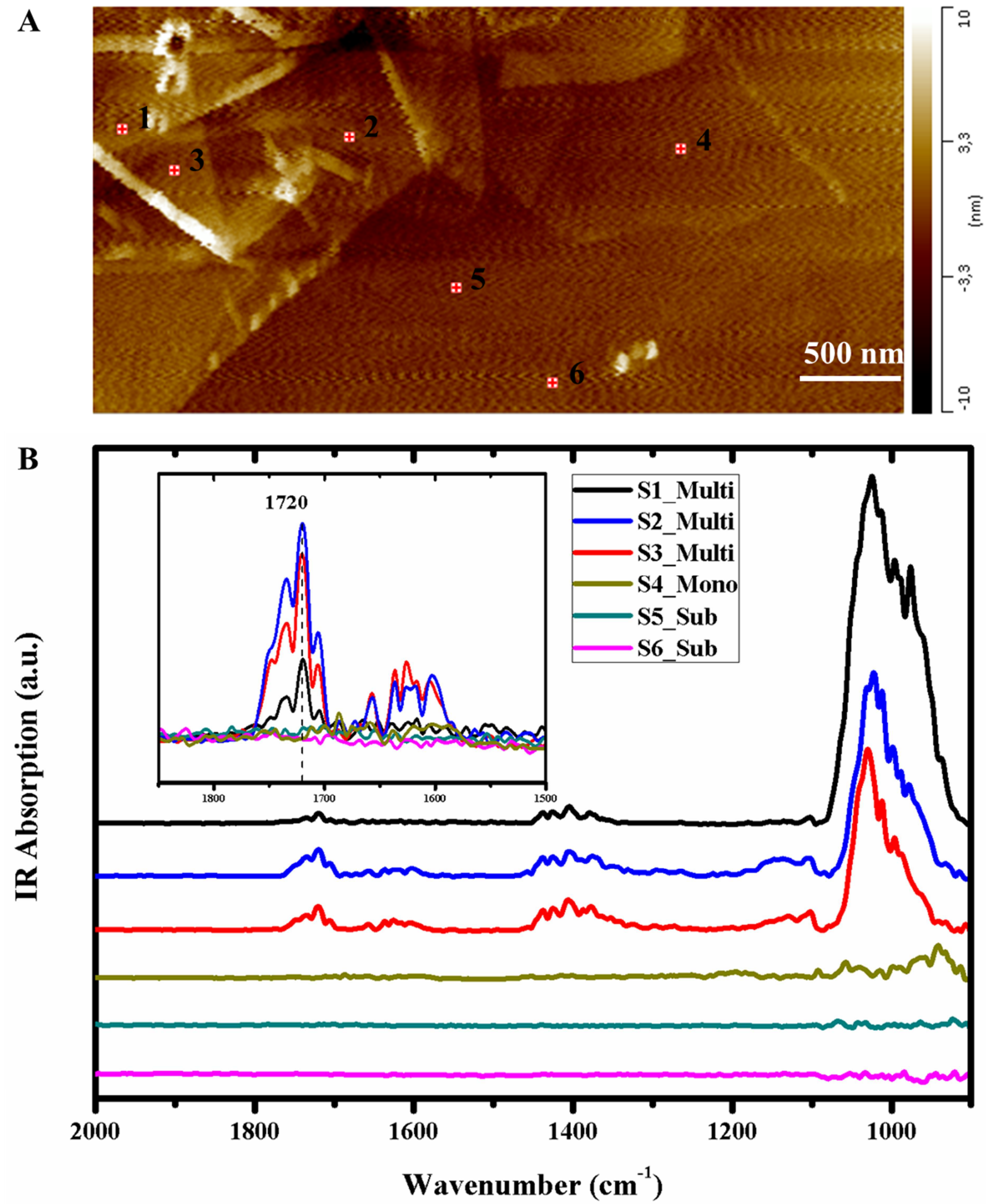

Fig. 1. (A) AFM topography image $(4 \mu \mathrm{m} \times 2 \mu \mathrm{m})$ of GO flakes adhering to a gold substrate. Lateral resolution is $\sim 40 \mathrm{~nm}$; the wavy lines are an artefact. In the upper left corner are two holes that were burned in the sample during optimization of laser power. (B) AFM-IR absorption spectra from the $900-2000 \mathrm{~cm}^{-1}$ range. The inset is an enlargement of the spectra in the $1500-1850 \mathrm{~cm}^{-1}$ range. Spectra S1-S6 were recorded at the sites indicated by red markers in the AFM image, on multilayers (Multi), monolayer (Mono) and substrate (Sub). Wavenumber resolution was set to $4 \mathrm{~cm}^{-1} / \mathrm{pt}$. 
In traditional FTIR experiments on oxidized graphite and graphene, IR absorption in the 970$1180 \mathrm{~cm}^{-1}$ region indicates stretches of $\mathrm{C}-\mathrm{O}$, such as are present in a number of molecular structures, such as epoxy, hydroxyl, ether and carboxyl. ${ }^{[12,14-16,27]}$ Depending on the experimental setup, absorption in the $970-1100 \mathrm{~cm}^{-1}$ range has been assigned to epoxy. ${ }^{[16,27-29]}$ Absorption from 900-1100 $\mathrm{cm}^{-1}$, with a maximum peak at $1025 \mathrm{~cm}^{-1}$, observed for the multilayer spectra (Fig. 1B), is consistent with a mixture of C-O stretching vibrations, mainly from a configuration resembling epoxy. The strength of the C-O peaks relative to the others suggests highest density for oxygen bonded singly to carbon, in good agreement with our XPS results (Supplementary Note 1 and Fig. S2) and with other published work. ${ }^{[30,31]}$

The absorption band observed at $1300-1450 \mathrm{~cm}^{-1}$ in Fig. 1B could originate from hydroxyl ${ }^{[12]}$ attached to aromatic carbon that is situated above the carbon plane, giving rise to deformation vibrations, ${ }^{[32]}$ or from hydroxyl in carboxyl configuration, absorbing in bending mode. ${ }^{[33]}$ The inset of Fig. 1B shows the IR absorption profiles in the $1500-1850 \mathrm{~cm}^{-1}$ range in more detail. In the multilayer spectra (S2 and S3), absorption at $\sim 1600 \mathrm{~cm}^{-1}$ could result from the aromatic bond stretching in the carbon plane. This peak is weak in the multilayer spectrum of S1 but it is consistent with high absorption for the oxidized GO, suggesting that the number of double bonds in the carbon network is lower at the expense of more oxidized groups at this site. The absorption region at $1720 \mathrm{~cm}^{-1}$ is characteristic of $\mathrm{C}=\mathrm{O}$ stretching vibrations from carbonyl. However, it is difficult to distinguish different carbonyls, such as carboxyls, ketones, lactones and anhydride due to the overlapping spectral features $\left(1650-1850 \mathrm{~cm}^{-1}\right)$ for their vibrations. ${ }^{[15,34-36]}$ Spectrum S4 was recorded on a single layer GO that was only $1 \mathrm{~nm}$ thick. Absorption in the $900-1100 \mathrm{~cm}^{-1}$ range is much lower in monolayer than the absorption for multilayer GO. Higher frequency absorption is negligible but the sample is only 1 molecular layer of carbon with associated oxygen. In the control spectra, S5 and S6, there were no distinct IR peaks. The assignments of main peaks in the AFM-IR were consistent with our 
$\mathbf{A}$
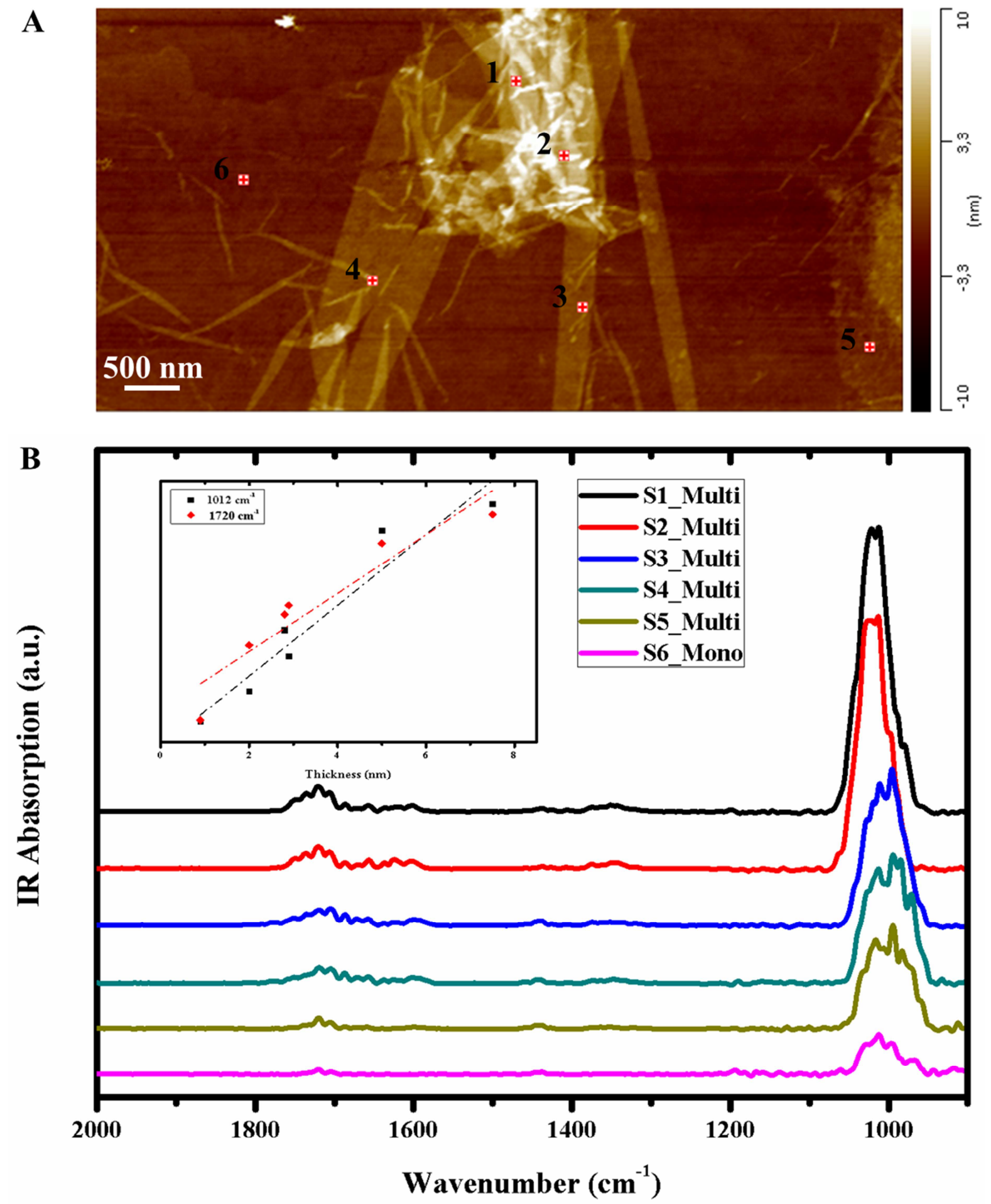

Fig. 2. (A) AFM topography image $(8 \mu \mathrm{m} \times 4 \mu \mathrm{m})$ of GO flakes on a gold substrate. (B) AFM-IR absorption spectra in the $900-2000 \mathrm{~cm}^{-1}$ range. The inset shows the IR absorption vs. the thickness of samples at two wavenumbers: 1012 and $1720 \mathrm{~cm}^{-1}$. Spectra S1-S6 were recorded at the positions indicated by red markers on the AFM image. Wavenumber resolution was set to $4 \mathrm{~cm}^{-1} / \mathrm{pt}$. 
micro-FTIR spectra in Supplementary Fig. S3 but with small shifts of the band for $\mathrm{C}=\mathrm{O}$ stretching vibrations at $\sim 1700 \mathrm{~cm}^{-1}$, $-\mathrm{OH}$ at $\sim 1500 \mathrm{~cm}^{-1}$, C-O at $\sim 1100 \mathrm{~cm}^{-1}$. A Raman spectrum of the GO displayed a spectrum consistent with the structural imperfections introduced to the graphene by the various ways oxygen was attached (Supplementary Note 2 and Fig. S4).

We proved reproducibility by repeating the experiment with new tips and new samples. In some cases, we found new relationships. Figure 2, from a new experiment, shows that absorption spectra mainly reproduced the data of Fig. 1, with the same main absorption in the $900-1100 \mathrm{~cm}^{-1}$ range but for this sample, there is a slight shift for the maximum peak from 1025 to $1012 \mathrm{~cm}^{-1}$. This suggests a slight difference in the proportion of C-O bonds beneath the tip, causing a slight shift in the absorption profile. Absorption at $1720 \mathrm{~cm}^{-1}$, assigned to $\mathrm{C}=\mathrm{O}$, is also observed in Fig. 2. Comparison of the single layer spectrum in Fig. 1 (S4) and Fig. 2 (S6) shows intensity differences in the $900-1100 \mathrm{~cm}^{-1}$ range. Absorption at $1720 \mathrm{~cm}^{-1}$ for Fig. 1 (S4) is nearly not visible, which could result from small differences in tip sensitivity or from different molecular structure on the sample but most likely, it is the result of differences in the distribution of the oxide groups. Lack of absorption at $1720 \mathrm{~cm}^{-1}$ is consistent with a low concentration of $\mathrm{C}=\mathrm{O}$ in the monolayer, as can also be seen in Fig. 3A, where the IR absorption map at $1720 \mathrm{~cm}^{-1}$ shows lower absorption over the monolayer than for the neighbouring multilayers. This could imply that the multilayers contain more carbonyl groups than the monolayer in this case, or the number of carbonyl groups over monolayer is below the detection limit of AFM-IR. By combining the information from the spectra in Figs $1 \mathrm{~B}$ and $2 \mathrm{~B}$, we get a good perspective of the fingerprint region, with evident characteristic peaks for C-O. It is even possible to identify slight absorption shifts for specific positions, such as the C-O peaks from S2 and S3 in Fig. 2B. These cannot be resolved with standard IR. ${ }^{[14,15]}$ 
The inset of Fig. 2B shows that IR absorption increases proportionally with sample thickness for absorption at $1012 \mathrm{~cm}^{-1}$ and $1720 \mathrm{~cm}^{-1}$. For each additional single layer of GO, IR absorption intensity increases by almost the same amount (i.e. 2 times relative to the value for a monolayer). Small variations most likely result from local variation in the GO structure or the type and density of oxygen bonding. The growth of intensity with the double of a monolayer suggests that monolayers are not homogeneous and have lower oxidation levels. thickness dependent IR absorption has previously been observed in attenuated total reflectance AFM-IR, using an inverted microscope with laser excitation from below. ${ }^{[37]}$

We further scanned other wavelengths to explore for other bonds. C-H stretches in the 2500$3600 \mathrm{~cm}^{-1}$ range are shown in Supplementary Fig. S5 for the same six sites as in Fig. 2A. Absorption at $2854 \mathrm{~cm}^{-1}$ and $2924 \mathrm{~cm}^{-1}$ represent $\mathrm{C}-\mathrm{H}$ stretching in aliphatic and aromatic groups. We did not detect any IR absorption in the $3000-3600 \mathrm{~cm}^{-1}$ range where hydroxyl stretching is expected ${ }^{[27,33]}$ such as would indicate adsorbed water and carboxyl groups. This is consistent with very little or no water associated with GO surfaces, which also agrees with the micro-FTIR spectra of few-layer GO shown in Supplementary Fig. S3 where vibration band from water could not be detected either. With FTIR experiments on bulk GO of Supplementary Fig. S6, we can find a peak around $3400 \mathrm{~cm}^{-1}$ corresponding to hydroxyl stretching, which might originate from water in the bulk. Except for one spectrum, we found a similar relationship as $\mathrm{C}-\mathrm{O}$ and $\mathrm{C}=\mathrm{O}$ bonds between IR absorption and $\mathrm{GO}$ thickness for $\mathrm{C}$ H groups, as shown by the inset of Supplementary Fig. S5. The variation of absorption intensity for the C-H peak is likely a result of its heterogeneous distribution or orientation of this bond.

An IR absorption map generated from the GO flakes of Fig. 1, at wavenumber $1720 \mathrm{~cm}^{-1}$, is shown in Fig. 3A. This represents $\mathrm{C}=\mathrm{O}$ stretches and is confined to the $\mathrm{GO}$ flakes. It is 
strongest along edges and folds in the multilayer but in the monolayer, absorption is more patchy so either the carbonyl groups are not present in some areas in the monolayer or the absorption is below the detection limit. It is more likely that carbonyl density varies locally than that detection is limited because absorption is clear along a fold in a monolayer (Dashed Line 2). As expected, no $\mathrm{C}=\mathrm{O}$ absorption is observed on the bare gold surface.
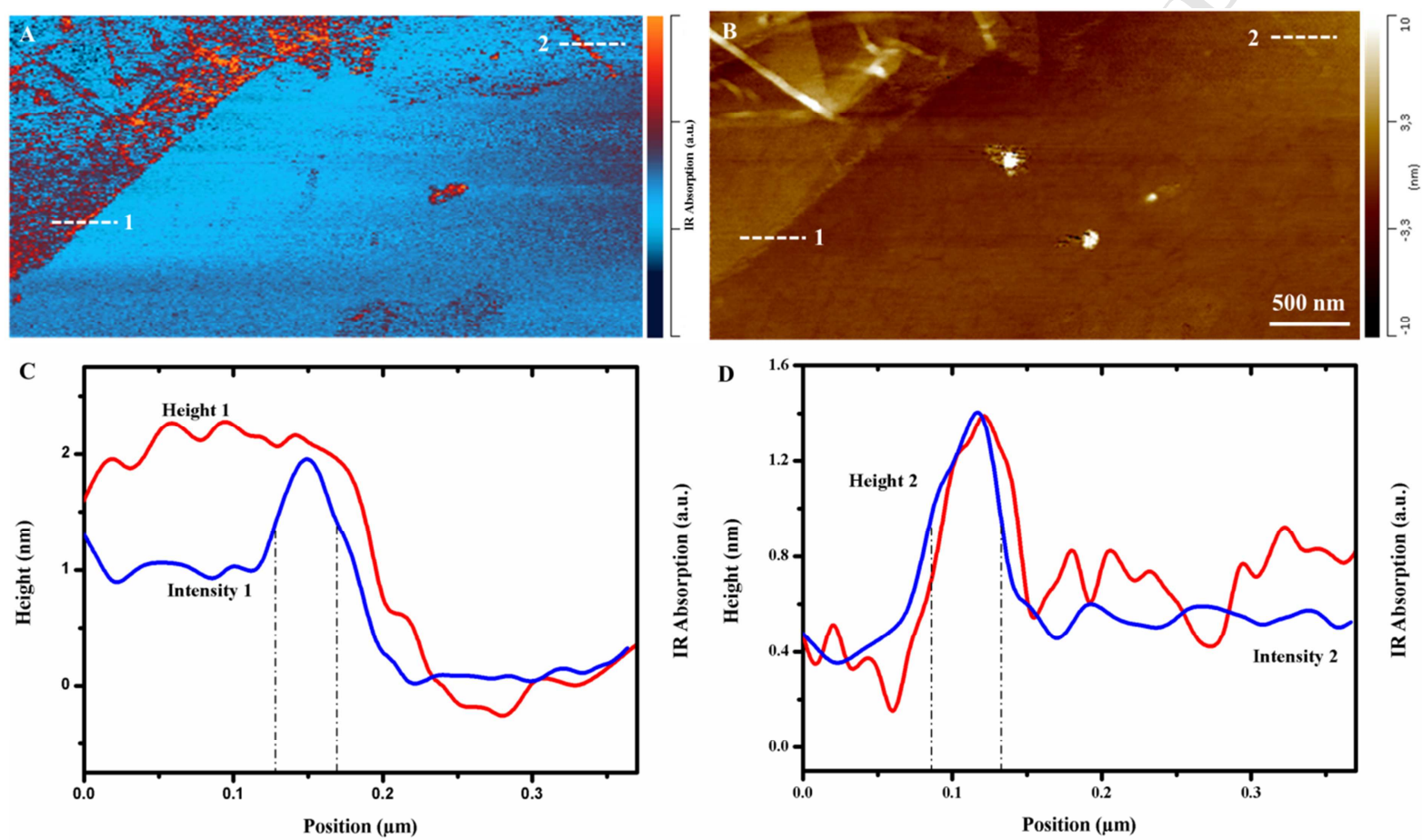

Fig. 3. (A) The GO IR absorption map collected at $1720 \mathrm{~cm}^{-1}$, which represents the $\mathrm{C}=\mathrm{O}$ stretch and (B) the AFM topography image from the same location. Feature height and IR absorption profiles were recorded along stipulated line marked by $1(\mathbf{C})$ and marked by $2(\mathbf{D})$.

Our results are consistent with the $\mathrm{LK}$ model for $\mathrm{GO},{ }^{[7]}$ i.e. $\mathrm{C}=\mathrm{O}$ terminate the flake edges, but our results provide the justification to significantly extend from that model. They demonstrate that $\mathrm{C}=\mathrm{O}$ is not only confined to the edges. From XPS spectra, it is clear that carbon atoms, in a carboxyl arrangement, make up a significant proportion of total carbon, $8.5 \%$ in our work. The low ratio of edge to plane carbon atoms in $\mathrm{GO}$ means that $\mathrm{C}=\mathrm{O}$ must not sit only on edges. The map in Fig. 3A clearly demonstrates this. IR absorption is high along the edges and folds and also on the planes, in discrete domains on the multilayer GO, 
such as in the upper left of the map. A large number of small $\mathrm{C}=\mathrm{O}$ domains, in the range of tens of nanometres, are visible on the basal plane of multilayer GO but such domains are less distinct on single layer GO. They are visible but are distributed, as we see on the small patch of a single layer at the lower and upper right side of the map of Fig. 3A.

To show how absorption varies over edges and folds of GO, we made cross sections, with absorption and height profiles (Fig. 3C and D) at the locations indicated in Fig. 3A and B. Fig. $3 \mathrm{C}$ shows that GO thickness is $\sim 2 \mathrm{~nm}$, i.e. a GO double layer. Absorption was strongest at the edge, where it was twice that of the single layer, but it was not zero on the plane, indicating $\mathrm{C}=\mathrm{O}$ presence there as well. The profile in Fig. 3D was taken across a fold in the single layer GO. Absorption intensity is directly proportional to thickness but it does not scale linearly, suggesting higher density of $\mathrm{C}=\mathrm{O}$ on the folding areas than on single graphene layers. The absorption and height profiles (Fig. 3C, D) can also be used to estimate the lateral resolution of AFM-IR on single and double layers of GO. Deduced from the absorption intensity curve, we found lateral resolution to be $\sim 40 \mathrm{~nm}$ in this case. In comparison, the lateral resolution of conventional FTIR is always limited by the optical diffraction of the IR wavelength, which is several micrometres. ${ }^{[38]}$ This is an important point. The AFM-IR technique improves spatial resolution by two orders of magnitude.

Figure 4 shows absorption maps for the wavenumber consistent with $\mathrm{C}-\mathrm{O}\left(1065 \mathrm{~cm}^{-1}\right)$ and $\mathrm{C}=\mathrm{O}\left(1720 \mathrm{~cm}^{-1}\right)$ stretches for the same set of GO flakes. Comparison of absorption on the plane and along edges and over folds in the multilayer (on the right side) shows strong intensity for $\mathrm{C}-\mathrm{O}$ on the plane and weaker intensity on some edges and folds. $\mathrm{C}=\mathrm{O}$ absorption was strong over the planes and stronger along the edges where $\mathrm{C}-\mathrm{O}$ absorption was weak, suggesting increased $\mathrm{C}=\mathrm{O}$ bond density at the expense of $\mathrm{C}-\mathrm{O}$ bonds on the edges. The small flake attached to the far left of the multilayer is a monolayer. Here the absorption pattern 


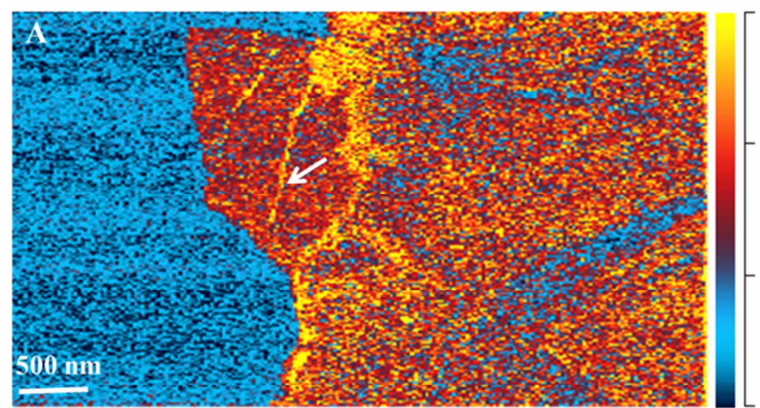

C-O mapping

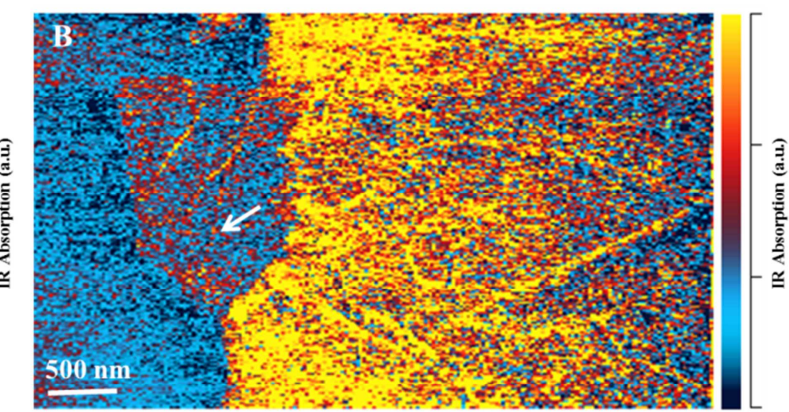

$\mathrm{C}=\mathbf{O}$ mapping

Fig. 4. The IR absorption map from the same site at wavenumber (A) $1065 \mathrm{~cm}^{-1}$, which represents the $\mathrm{C}-\mathrm{O}$ stretch and $(\mathbf{B})$ at $1720 \mathrm{~cm}^{-1}$, the $\mathrm{C}=\mathrm{O}$ stretch. The intensity in the area of the arrow suggests different distributions of $\mathrm{C}-\mathrm{O}$ and $\mathrm{C}=\mathrm{O}$ bonds along folds.

suggests a dense coverage of $\mathrm{C}-\mathrm{O}$ on the plane with increased density on some folds, while $\mathrm{C}=\mathrm{O}$ is mainly concentrated on edges and folds where $\mathrm{C}-\mathrm{O}$ is less abundant (arrow). In the plane of the single $\mathrm{GO}$ layer, the $\mathrm{C}=\mathrm{O}$ absorption domains were much weaker than those of $\mathrm{C}$-O. In the $\mathrm{GO}$ multilayers, $\mathrm{C}=\mathrm{O}$ absorption domains were stronger on the plane and on some folds than on single GO layers. The effect of oxygen containing functional groups and defects on the folding and wrinkling behaviors can be important. The pristine graphene become intrinsically stable through wrinkling itself with an amplitude of about $0.1 \mathrm{~nm}$ and minimizing the total free energy by thermal fluctuation. ${ }^{[39]}$ Apart from intrinsic wrinkles of graphene, the out-of-plane wrinkling has been observed by topological defect sites, where high elastic energy is relaxed by distorted lattices. ${ }^{[40]}$ Upon adding one Stone-Wales defect in graphene, the local wrinkle is with an augmented amplitude of about $0.3 \mathrm{~nm}$, while a row of Stone-Wales defects leads to a larger wrinkle of about $0.5 \mathrm{~nm}$ that resembles to folds. ${ }^{[41]}$ Accordingly, graphene could wrinkle and fold easily if more topological defect sites exist. Once the oxygens in the form of epoxy, hydroxyl and carboxyl groups attach to the graphene surface, the bonded carbon atoms will be pulled away from the original plane and the local carbon bond length enlarges. It induces the conversion of oxidized carbon from in-plane $\mathrm{sp}^{2}$ hybridization to tetrahedral $\mathrm{sp}^{3}$ hybridization, which makes graphene much easier to wrinkle 
and fold. With same degree of functionality, carboxyl groups show a smaller shear stress than hydroxyls, indicating a higher degree of wrinkling. ${ }^{[42]}$ In this picture oxygen functional groups concentrate at defective sites, leading to the formation of folds in GO this is consistent with our observations in the AFM-IR where the more oxygen functional groups preferentially sitting along folds and edges.

We built an updated structural model for GO on the base of the LK model, ${ }^{[7]}$ so it would describe published data as well as the new results from high resolution IR imaging, namely, the presence of $\mathrm{C}=\mathrm{O}$ on the eadge and plane. Although previous Scholz-Boehm ${ }^{[43]}$ and Szabo-Dekany (SD) ${ }^{[44]}$ models assumed the presence of $\mathrm{C}=\mathrm{O}$ on basal planes of GO, there no carboxyls and epoxies present in the model, which was excluded by Casabianca et al. ${ }^{[9]}$ From our experiments, we confirm parts of earlier proposed models that $\mathrm{C}=\mathrm{O}$ on its edge (LK model $)^{[7]}$ and plane $(\mathrm{SD} \text { model })^{[43]}$. Therefore, we propose an updated structural model for GO. The spatial distribution of oxygen and the newly discovered bonding relationships is shown in Fig. 5. The new model fits a number of criteria. (1) Morphology. There is significant structural disorder in the GO plane to accommodate a variety of oxygen bonding configurations. (2) Chemical composition. C-O dominates, mainly in configurations resembling hydroxyl and epoxy, with some ether; $\mathrm{C}=\mathrm{O}$, is present as carboxyl and ketone. Lactone and anhydride ${ }^{[35,45]}$ which are minor, can be considered to be combinations of ketone and ether. (3) Spatial distribution. C-O is mostly on the plane (Domain I, green); $\mathrm{C}=\mathrm{O}$ is present on the planes but is more abundant on edges and folds (Domain II with vacancy defects, blue). In the LK model, carboxyl is present only at edges. Ketone groups in defective sites of $\mathrm{C}-\mathrm{C}$ bonds are proposed in SD model and it is also present in edges of $\mathrm{GO}^{[16]}$. Because of the abundance of available binding sites, $\mathrm{C}-\mathrm{O}$ and $\mathrm{C}=\mathrm{O}$ bonds are common on edges (Edge III, orange). Lactone and anhydride can also be decorated at the periphery of GO sheet. ${ }^{[8,35]}$ 
A

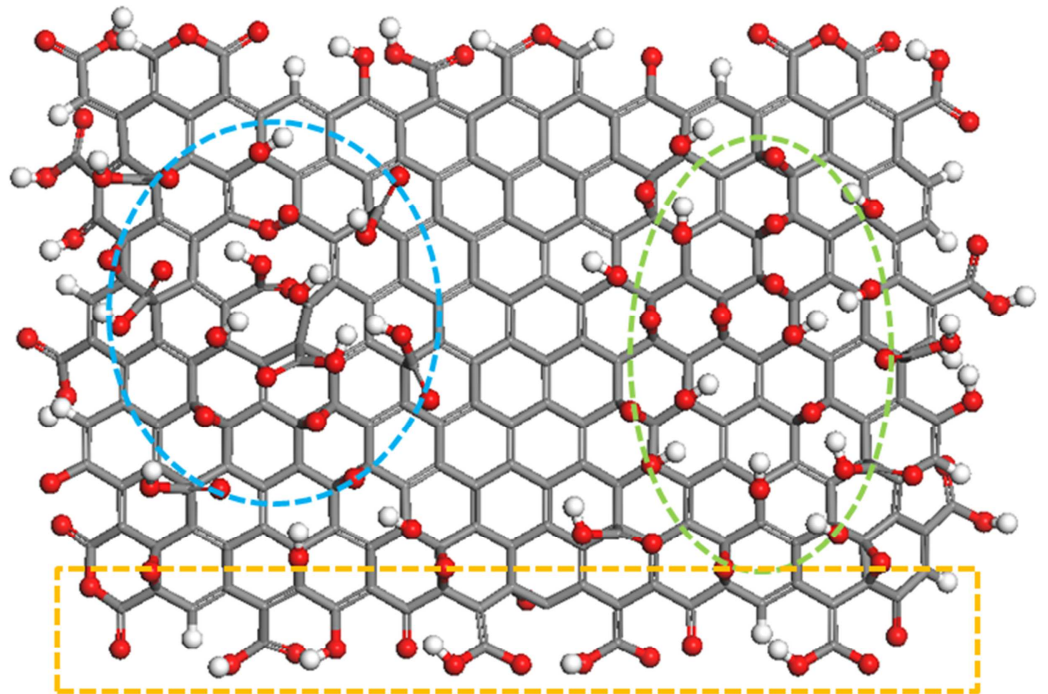

Top view

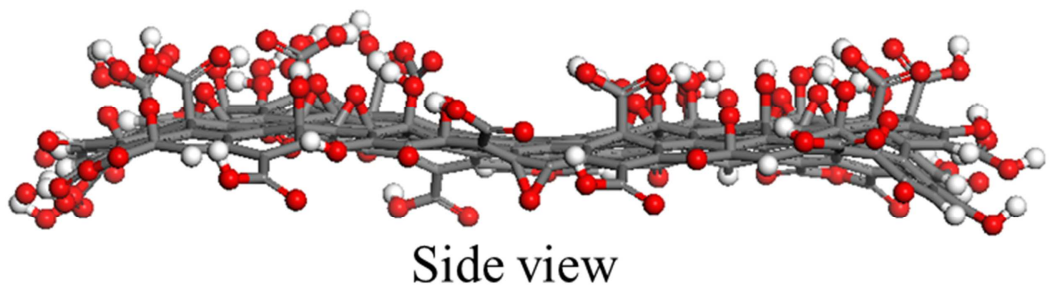

B
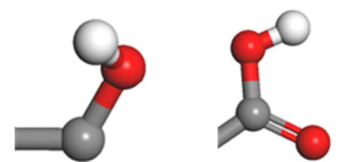

Alcohol

Carboxyl
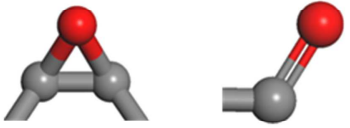

Epoxide

Ketone

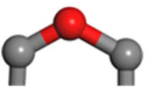

Ether

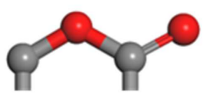

Lactone

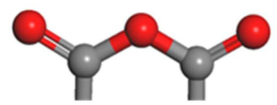

Anhydride

Fig. 5. (A) New structural model of GO in top and side views. Domain I (green) shows the distribution of $\mathrm{C}-\mathrm{O}$ and Domain II (blue) shows vacancies with possible $\mathrm{C}=\mathrm{O}$ distributions, Edge III (orange) shows examples of possible $\mathrm{C}-\mathrm{O}$ and $\mathrm{C}=\mathrm{O}$ groups. $(\mathbf{B})$ The oxygen configurations that are possible on GO. Grey, red and white balls represent $\mathrm{C}, \mathrm{O}$ and $\mathrm{H}$ atoms.

The updated model describes the observed oxygen distribution, especially the presence of $\mathrm{C}=\mathrm{O}$ on the interior and edge of GO. There are many ways that this can come about. A recent TEM study offered evidence of holes in the GO sheet. ${ }^{[17]}$ Abundant defects in GO, either present initially or promoted by reaction with oxygen, provide sites for $\mathrm{C}=\mathrm{O}$ formation. Carbonyl pairs form where C-C bonds are broken. ${ }^{[44,46]}$ Another possibility is on folds, where higher strain in the bonds makes them easy to cleave and the extra space near neighbouring $\mathrm{C}$ atoms leads to $\mathrm{C}-\mathrm{O}$ and $\mathrm{C}=\mathrm{O}$ bond formation.

\section{Conclusion}


In summary, we have demonstrated that for single and multilayer GO, the spatial distribution of $\mathrm{C}-\mathrm{O}$ and $\mathrm{C}=\mathrm{O}$ can be determined using AFM-IR, with resolution on the scale of a few tens of nanometres. From IR spectra at specific positions on GO, we observed C-O stretching at $900-1100 \mathrm{~cm}^{-1}$, mainly from epoxy; at $1300-1450 \mathrm{~cm}^{-1}$, from hydroxyl in various vibration modes; $\mathrm{C}=\mathrm{C}$ stretching at $1600 \mathrm{~cm}^{-1}$ and $\mathrm{C}=\mathrm{O}$ stretching at $1720 \mathrm{~cm}^{-1}$ from carbonyl. The intensity of the peaks at $1012 \mathrm{~cm}^{-1}(\mathrm{C}-\mathrm{O})$ and $1720 \mathrm{~cm}^{-1}(\mathrm{C}=\mathrm{O})$ is proportional to GO layer thickness, indicating that each layer is oxidized individually. The ultrahigh spatial resolution that is possible with IR mapping (better than $40 \mathrm{~nm}$ ) shows oxygen in discrete domains in the plane, on the edges and over areas where the GO is folded. The new ability to determine the type and position of oxygen bonding from precise imaging offers the possibility to link nanoscale structure with function, paving the way for targeted tethering of ions, polymers and biomaterials.

\section{Acknowledgments}

We thank the NanoGeoScience group members, especially J. Generosi and J. Matthiesen for helpful discussions. We acknowledge support from the Innovation Fund Denmark (NIAGRA, DAGATE) and the Danish Council for Independent Research (DFF-6108-00681) "Getting in Contact with Molecules”. Z.L. Liu acknowledges the financial support from the NanoGeoSceince group and the China Scholarship Council (CSC).

\section{Appendix A. Supplementary data}

The following is the supplementary data related to this article. 


\section{References}

[1] D. R. Dreyer, A. D. Todd, C. W. Bielawski, Harnessing the chemistry of graphene oxide. Chem. Soc. Rev., 43(2014), 5288-5301.

[2] K. P. Loh, Q. Bao, G. Eda, M. Chhowalla, Graphene oxide as a chemically tunable platform for optical applications. Nat. Chem., 2(2010), 1015-1024.

[3] Q. Zheng, Z. Li, J. Yang, J. -K. Kim, Graphene oxide-based transparent conductive films. Prog. Mater. Sci., 64(2014), 200-247.

[4] D. S. Su, S. Perathoner, G. Centi, Nanocarbons for the Development of Advanced Catalysts. Chem. Rev., 113(2013), 5782-5816.

[5] V. Georgakilas, M. Otyepka, A. B. Bourlinos, V. Chandra, N. Kim, K. C. Kemp, et al. Functionalization of graphene: covalent and non-covalent approaches, derivatives and applications. Chem. Rev., 112(2012), 6156-6214.

[6] K. Yang, L. Feng, X. Shi, Z. Liu, Nano-graphene in biomedicine: theranostic applications. Chem. Soc. Rev., 42(2013), 530-547.

[7] A. Lerf, H. He, M. Forster, J. Klinowski, Structure of graphite oxide revisited. J. Phys. Chem. B, 102(1998), 4477-4482.

[8] W. Gao, L. B. Alemany, L. Ci, P. M. Ajayan, New insights into the structure and reduction of graphite oxide. Nat. Chem., 1(2009), 403-408.

[9] L. B. Casabianca, M. A. Shaibat, W. W. Cai, S. Park, R. Piner, R. S. Ruoff, Y. Ishii, NMR-based structural modeling of graphite oxide using multidimensional ${ }^{13} \mathrm{C}$ solid-state NMR and ab initio chemical shift calculations. J. Am. Chem. Soc., 132(2010), 5672-5676.

[10] L. R. De Jesus, R. V. Dennis, S. W. Depner, C. Jaye, D. A. Fischer, S. Banerjee, Inside and outside: X-ray absorption spectroscopy mapping of chemical domains in graphene oxide. J. Phys. Chem. Lett., 4(2013), 3144-3151. 
[11] K. N. Kudin, B. Ozbas, H. C. Schniepp, R. K. Prud'Homme, I. A. Aksay, R. Car, Raman spectra of graphite oxide and functionalized graphene sheets. Nano Lett., 8(2008), 36-41.

[12] K. Krishnamoorthy, M. Veerapandian, K. Yun, S.-J. Kim, The chemical and structural analysis of graphene oxide with different degrees of oxidation. Carbon, 53(2013), 38-49.

[13] D. Zhan, Z. Ni, W. Chen, L. Sun, Z. Luo, L. Lai, et al. Electronic structure of graphite oxide and thermally reduced graphite oxide. Carbon, 49(2011), 1362-1366.

[14] M. Acik, C. Mattevi, C. Gong, G. Lee, K. Cho, M. Chhowalla, Y. J. Chabal, The role of intercalated water in multilayered graphene oxide. ACS Nano, 4(2010), 5861-5868.

[15] M. Acik, G. Lee, C. Mattevi, A. Pirkle, R. M. Wallace, M. Chhowalla, et al. The role of oxygen during thermal reduction of graphene oxide studied by infrared absorption spectroscopy. J. Phys. Chem. C, 115(2011), 19761-19781.

[16] D. Lee, L. De Los Santos V, J. Seo, L. L. Felix, A. Bustamante D, J. Cole, C. Barnes, The structure of graphite oxide: investigation of its surface chemical groups. J. Phys. Chem. B, 114(2010), 5723-5728.

[17] K. Erickson, R. Erni, Z. Lee, N. Alem, W. Gannett, A. Zettl, Determination of the local chemical structure of graphene oxide and reduced graphene oxide. Adv. Mater. 22(2010), 4467-4472.

[18] K. A. Mkhoyan, A. W. Contryman, J. Silcox, D. A. Stewart, G. Eda, C. Mattevi, et al. Atomic and electronic structure of graphene-oxide. Nano Lett., 9(2009), 1058-1063.

[19] A. Tararan, A. Zobelli, A. M. Benito, W. K. Maser, O. Stephan, Revisiting Graphene Oxide Chemistry via Spatially-Resolved Electron Energy Loss Spectroscopy. Chem. Mater., 28(2016), 3741-3748.

[20] A. Dazzi, C. B. Prater, AFM-IR: Technology and Applications in Nanoscale Infrared Spectroscopy and Chemical Imaging. Chem. Rev., 117 (2017), 5146-5173. 
[21] A. Centrone, Infrared Imaging and Spectroscopy Beyond the Diffraction Limit. Annu. Rev. Anal. Chem., 8(2015), 101-126.

[22] A. Dazzi, C. B. Prater, Q. C. Hu, D. B. Chase, J. F. Rabolt, C. Marcott, AFM-IR: Combining Atomic Force Microscopy and Infrared Spectroscopy for Nanoscale Chemical Characterization. Appl. Spectrosc., 66(2012), 1365-1384.

[23] W. S. Hummers Jr, R. E. Offeman, Preparation of graphitic oxide. J. Am. Chem. Soc. 80(1958), 1339-1339.

[24] S. Eigler, M. Enzelberger-Heim, S. Grimm, P. Hofmann, W. Kroener, A. Geworski, et al. Wet chemical synthesis of graphene. Adv. Mater., 25(2013), 3583-3587.

[25] A. Naumov, F. Grote, M. Overgaard, A. Roth, C. E. Halbig, K. Nørgaard, et al. Graphene Oxide: A One-versus Two-Component Material. J. Am. Chem. Soc., 138(2016), $11445-11448$.

[26] N. Bovet, M. Yang, M. S. Javadi, S. L. S. Stipp, Interaction of alcohols with the calcite surface. Phys. Chem. Chem. Phy., 17(2015), 3490-3496.

[27] T. F. Yeh, J. M. Syu, C. Cheng, T. H. Chang, H. Teng, Graphite oxide as a photocatalyst for hydrogen production from water. Adv. Func. Mater., 20(2010), 2255-2262.

[28] J. Guerrero-Contreras, F. Caballero-Briones, Graphene oxide powders with different oxidation degree, prepared by synthesis variations of the Hummers method. Mater. Chem. Phys., 153(2015), 209-220.

[29] A. Gharib, L. Vojdanifard, N. N. Pesyan, M. Roshani, A New Application of NanoGraphene Oxide (NGO) as a Heterogeneous Catalyst in Oxidation of Alcohols Types. Chemistry, 1(2015), 151-158.

[30] T. Taniguchi, S. Kurihara, H. Tateishi, K. Hatakeyama, M. Koinuma, H. Yokoi, et al. pH-driven, reversible epoxy ring opening/closing in graphene oxide. Carbon, 84(2015), 560566. 
[31] H. Jeong, M. Jin, K. So, S. Lim, Y. Lee, Tailoring the characteristics of graphite oxides by different oxidation times. J. Phys. D: Appli. Phys., 42(2009), 065418.

[32] J. Zhang, H. Yang, G. Shen, P. Cheng, J. Zhang, S. Guo, Reduction of graphene oxide via L-ascorbic acid. Chem. Commun., 46(2010), 1112-1114.

[33] J. H. Wang, S. Liang, L. Ma, S. J. Ding, X. F. Yu, L. Zhou, Q. Q. Wang, One-pot synthesis of CdS-reduced graphene oxide 3D composites with enhanced photocatalytic properties. CrystEngComm., 16(2014), 399-405.

[34] E. Fuente, J. A. Menéndez,M. A. Díez, D. Suárez, M. A. Montes-Morán, Infrared spectroscopy of carbon materials: a quantum chemical study of model compounds. J. Phys. Chem. B, 107 (2003), 6350-6359.

[35] C. Su, M. Acik, K. Takai, J. Lu, S. Hao, Y Zheng., Probing the catalytic activity of porous graphene oxide and the origin of this behavior. Nat. Commun., 3(2012), 1298.

[36] H. Fu, X. Qu, W. Chen, D Zhu, Transformation and destabilization of graphene oxide in reducing aqueous solutions containing sulfide, Environ. Toxicol. Chem., 33(2014), 26472653.

[37] B. Lahiri, G. Holland, A. Centrone, Chemical imaging beyond the diffraction limit: Experimental validation of the PTIR technique. Small, 9(2013), 439-445.

[38] P. Lasch, D. Naumann, Spatial resolution in infrared microspectroscopic imaging of tissues. Biochim. Biophys. Acta, Biomembr., 1758(2006), 814-829.

[39] A. Fasolino, J. H. Los, M. I. Katsnelson. Intrinsic ripples in graphene. Nat Mater., 6(2007), 858-861.

[40] Y. Liu, B. I. Yakobson. Cones, pringles and grain boundary landscapes in graphene topology. Nano Lett., 10(2010), 2178-2183.

[41] X. Shen, X. Y. Lin, N. Yousefi, J. J. Jia, J. K. Kim. Wrinkling in graphene sheets and graphene oxide papers, Carbon, 66(2014), 84-92. 
[42] Q. B. Zheng, Y. Geng, S. J. Wang, Z. G. Li, J. K. Kim. Effects of functional groups on the mechanical and wrinkling properties of graphene sheets. Carbon, 48(2010), 4315-4322.

[43] W. Scholz, H. -P. Z. Boehm, Untersuchungen am graphitoxid. VI. Betrachtungen zur struktur des graphitoxids. Anorg. Allg. Chem., 1969, 369, 327-340.

[44] T. Szabó, O. Berkesi, P. Forgó, K. Josepovits, Y. Sanakis, D. Petridis, I. Dékány. Evolution of surface functional groups in a series of progressively oxidized graphite oxides. Chem. Mater., 18(2006), 2740-2749.

[45] L. Lai, L. Chen, D. Zhan, L. Sun, J. Liu, S. H. Lim, et al., One-step synthesis of $\mathrm{NH}_{2}-$ graphene from in situ graphene-oxide reduction and its improved electrochemical properties. Carbon, 49(2011), 3250-3257.

[46] Z. Li, W. Zhang, Y. Luo, J. Yang, J. G. Hou, How graphene is cut upon oxidation? J. Am. Chem. Soc., 131(2009), 6320-6321. 